\title{
Acoustic Properties of Sound Absorber from Modified Polyester with Filler Sodium Bicarbonate
}

\author{
NASMI HERLINA SARI ${ }^{1 *}$ and JAUHAR FAJRIN² \\ 'Department of Mechanical Engineering, Faculty of Engineering, Mataram University, \\ Jl. Majapahit No. 62 Mataram, Nusa Tenggara Barat Indonesia. \\ ${ }^{2}$ Department of Civil Engineering, Faculty of Engineering, Mataram University, \\ Jl. Majapahit No. 62 Mataram, Nusa Tenggara Barat Indonesia. \\ *Corresponding author E-mail: n.herlinasari@unram.ac.id \\ http://dx.doi.org/10.13005/ojc/3404061
}

(Received: July 10, 2018; Accepted: July 21, 2018)

\begin{abstract}
The combination of low price, ease of manufacturing and waterproofing characteristics has placed polyester resin as a potential sound absorbent material. Previous studies showed that adding filler material to the blending may increase the acoustics properties of a sound absorbent material. This study aims to investigate the potential of sodium bicarbonate to be employed as a filler to improve the acoustic properties of the sound absorber made of polyester resin. Two important acoustic parameters were carefully assessed; absorption coefficient and acoustic impedance. The results showed that the sound absorption performance increased significantly at low and medium frequencies in the presence of $\mathrm{NaHCO}_{3}$ filler in polyester resin. Meanwhile, the use of a back cavity on the absorbent material reduced the sound absorption performance of materials at low and medium frequencies. This suggests that sound absorber made of polyester with sodium bicarbonate filler may be used as an alternative for sound absorber materials.
\end{abstract}

Keywords: Acoustic properties, Filler, Impedance tube, Polyester, Sodium bicarbonate.

\section{INTRODUCTION}

The utilization of polymer material as a sound absorber has been an optinally and commercially valuable. In field application, a thermoset resin material such as polyester is not recommended for a sound absorber material due to its very low sound absorption coefficient, which is less than $0.2^{1}$. However, polyester resin has some advantages which include cheap, easily formed, corrosion resistant, and low water absorption².

In order to promote polymer for a sound absorber material, few researchers developed several methods to improve the acoustical properties of the polymer. Iwan et al., ${ }^{3}$ created a number of holes and configuration on the panel surface and investigated the changes in acoustics behavior

This is an Open Access article licensed under a Creative Commons Attribution-Non Commercial-Share Alike 4.0 International License (https://creativecommons.org/licenses/by-nc-sa/4.0/), which permits unrestricted Non Commercial use, distribution and reproduction in any medium, provided the original work is properly cited. 
due to both cavity and tube length. They reported that perforated panel (PP) achieved the best sound absorption coefficient at 0.186 in the frequency range of $522 \mathrm{~Hz}-700 \mathrm{~Hz}$, while the attachment of 18 tubes improved the sound absorption coefficient in the range of $0.248-0.286$ at the same frequency. Further, they stated that a great improvement of absorption coefficient was achieved when every perforation equipped with a short tube to form an array of constraint short thin tubes (ACST), which was 0.6 in the frequency range of $292-416 \mathrm{~Hz}$. In addition, Sari et al., ${ }^{1}$ investigated the acoustical properties of polyester resin mixed with corn husk fiber (CHF) and found that using more polyesters in the composite decreased the sound absorption coefficient. While in contrast, the increasing of corn husk fiber in the composite increased the sound absorption coefficient $(\alpha)$ of composites.

Furthermore, Xie et al., ${ }^{4}$ studied the characterization of a resonator perforated with flexible tubes and found that a larger cavity depth helped to get a better absorption coefficient in low frequency. They also reported that the thickness of the panel did not affect the absorption performance, and the longer penetration brought the greater peak absorption coefficient, but higher resonance frequency. In addition, Gheorghe ${ }^{5}$ created multi-layer micro-perforated structure panels and (MPP - porous - panel). Also, Chen et al., ${ }^{6}$ compared the acoustics properties of short ramie and ramie weaved reinforced composites panels with flame retardant ammonium polyphosphate (APP), plasticizer polybutylene adipate - co terephthalate (PBAT). They reported that the addition of flame retardant ammonium polyphosphate (APP), plasticizer polybutylene adipate - co - terephthalate (PBAT) had a positive effect on acoustic properties of PLLA/ramie fabric composites. Composites with a flame retardant were superior to composites without flame retardant. Jiang et al., ${ }^{7}$ investigated the sound absorption properties of chlorinated polyethylene composites (CPE) and seven hole polyester fiber (SHPE). They reported that fiber content, thickness, and depth cavity of the CPE/SHPF composites had significantly affected the acoustic properties. Acoustic absorption of the material increased with the increasing of SHPF content. A composite with $20 \%$ of SHPF content and $1 \mathrm{~mm}$ in thickness had an $\alpha$ value of 0.42 at a frequency range of $2500 \mathrm{~Hz}$. The maximum sound absorption coefficient was obtained at a thickness of $3 \mathrm{~mm}$, with a value of 0.695 at the frequency $2500 \mathrm{~Hz}$.

Sodium bicarbonate is found in nature and has a special molecular structure. When this material is added to the acid (i.e. milk, vinegar) and heated to a temperature of $70^{\circ} \mathrm{C}$ it will release carbon dioxide $\left(\mathrm{CO}_{2}\right)$. With this important characteristic, sodium bicarbonate which is currently widely used in industry such as effervescent powder may also be used in composites manufacturing. Although many investigations of using fillers in composites have been reported in the literature, the utilization of sodium hydroxide as a filler in a polymer composite to improve acoustic properties has never been reported. This article reports the experimental investigation of using sodium bicarbonate to improve the acoustic properties of the polyester resin.

\section{MATERIALS AND METHODS}

\section{Materials}

The characteristic of $\mathrm{NaHCO}_{3}$ powder is white color, non-toxic, biodegradable and eco-friendly ${ }^{8}$, with the following specifications; 99.0-100.5\% $\mathrm{NaHCO}_{3}$, $0.2 \%$ moisture and $\mathrm{pH}(10 \mathrm{~g} / \mathrm{L})$ of 8.6 . The polyester resin was obtained with a commercial type of 2250 BW-EX, which has a tensile strength of $8.8 \mathrm{~kg} / \mathrm{mm}^{2}$ and moduli strength of $500 \mathrm{~kg} / \mathrm{mm}^{2}$. Both materials, $\mathrm{NaHCO}_{3}$, and polyester are shown in Fig. $1 \mathrm{a}$ and $1 \mathrm{~b}$.

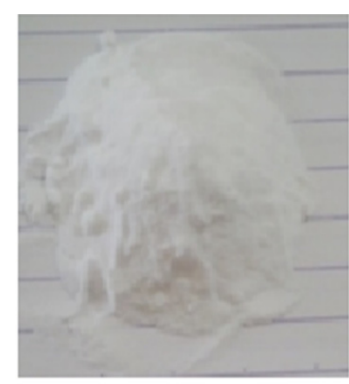

(a)

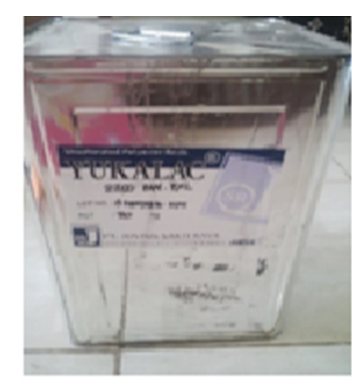

(b)
Fig. 1a. $\mathrm{NaHCO}_{3}$ and b. Polyester resin

\section{Samples Preparation}

The absorber materials were prepared by mixing polyester resin and $\mathrm{NaHCO}_{3}$ with a ratio of 30:70 (PN) dan 0:100 (RP) by volume fraction. The constituent materials were mixed and stirred using an impeller $500 \mathrm{rpm}$ for one minute. It was expected 
that theoretically, the addition of $\mathrm{NaHCO}_{3}$ will trigger the formation of gas bubbles which creates pores during the cooling and solidification process of the absorber materials.

There are two requirements that have to be ensured in order to provide high-quality foam; the first one is to ensure a uniform distribution of $\mathrm{NaHCO}_{3}$ in a matrix, and the second is to ensure the thermal characterization of $\mathrm{NaHCO}_{3}$ to avoid the formation of cracks prior to the solidification process. Having prepared the mixture by following the above two requirements, the mixture was then stored in a constant room temperature of $25^{\circ} \mathrm{C}$ to control initial drying rate before placing it in a microwave foaming for 1 minute. The microwave was set-up within intensity per unit mass of $12 \mathrm{~kW} / \mathrm{kg}$ at $2.4 \mathrm{GHz}$ at a temperature of $120^{\circ} \mathrm{C}$ for 20 seconds. The last step was demolding and finishing. The subsequent processes of preparing sound absorption material are depicted in Figures 2 and 3.

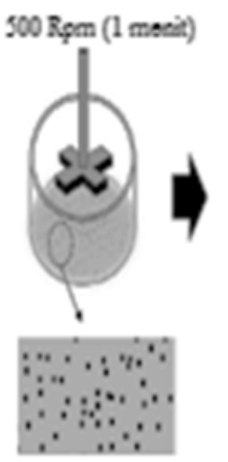

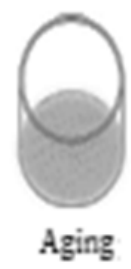

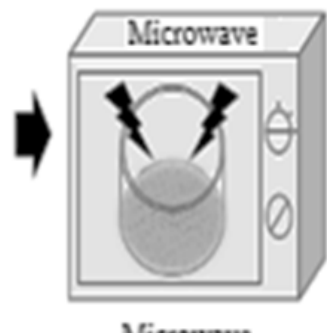

Microwave
Fig. 2. Manufacturing process of absorber materials

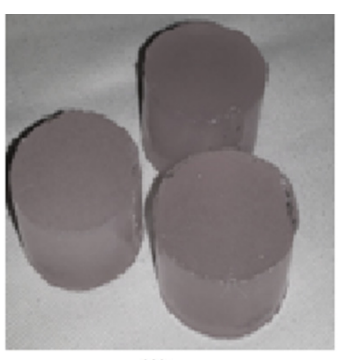

(A)

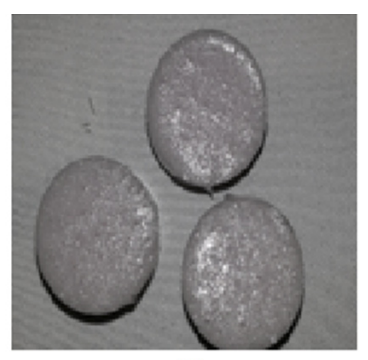

(B)
Fig. 3. Samples test of sound absorption properties a. RP, and b. PN

\section{Testing method}

The acoustic properties of the absorber samples were tested using impedance tubes of two microphones with the type of 4206 Brüel \& Kjær, as shown in Fig. 4. The samples were prepared in two thicknesses of $20 \mathrm{~mm}$ and $29 \mathrm{~mm}$, as per
ASTM E1050-98/ISO 10534-2. A small tube was prepared to measure acoustic parameters within the $100 \mathrm{kHz}-6400 \mathrm{~Hz}$ frequency range, while a loudspeaker was located on one end of the tube acting as the sound source. The test sample was placed at the other end to measure the absorption properties of the sound. Two Bruel \& Kjær microphones, type 4187 were positioned in front of the sample to record both sound absorption and sound reflection of the tested samples. Signals were recorded in terms of a function transferred between microphones. The collected data were further processed using a software provided by the testing machine supplier, Brüel \& Kjær, to obtain the sound absorption coefficients. Each sample was replicated three times to have the average value of the measurement.

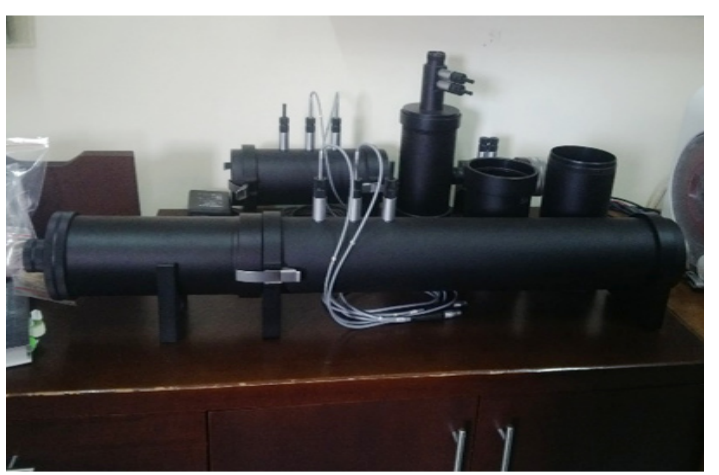

Fig. 4. Image photo of two microphones impedance tube (Type Bruel-Kjaer 1406)

\section{RESULTS AND DISCUSSIONS}

The results of the sound absorption measurement of all tested samples are graphically presented in Fig. 5. It is clearly shown that the peak sound absorption is reached within the frequency of $1300 \mathrm{~Hz}$ to $3100 \mathrm{~Hz}$. The PN sample has the highest absorption coefficient, which is nearly 1 . While, the maximum sound absorption coefficient of RP and RPcav are approximately 0.68 and 0.21 , respectively. These results indicate that the addition of $\mathrm{NaHCO}_{3}$ greatly improved the sound absorption performance of polyester resin. The improvement is most probably contributed by the presence of holes generated by $\mathrm{NaHCO}_{3}$ within the resin as a result of molecular opening due to the low compatibility reflection between $\mathrm{NaHCO}_{3}$ and the polyester resin. The presence of holes or cavities may convert the sound energy into thermal energy due to their friction with the molecules. This is also the reason why the 
sound absorption coefficient of sample PN at both low and high frequencies is higher than the others. For the panel with a thickness of $20 \mathrm{~mm}$, the absorption coefficient reached a maximum value of approximately 0.8 within a frequency range of $1000-6000 \mathrm{~Hz}$. This result is significantly higher than the value obtained by a perforated panel (PP) developed by lan et al., ${ }^{3}$ which showed that the perforated panel using 18 tubes had a sound absorption coefficient of $0.248-0.286$ within a frequency range of $522 \mathrm{~Hz}-700 \mathrm{~Hz}$. It proves that the $\mathrm{NaHCO}_{3}-$ polyester absorber can be a good alternative to the acoustic material.

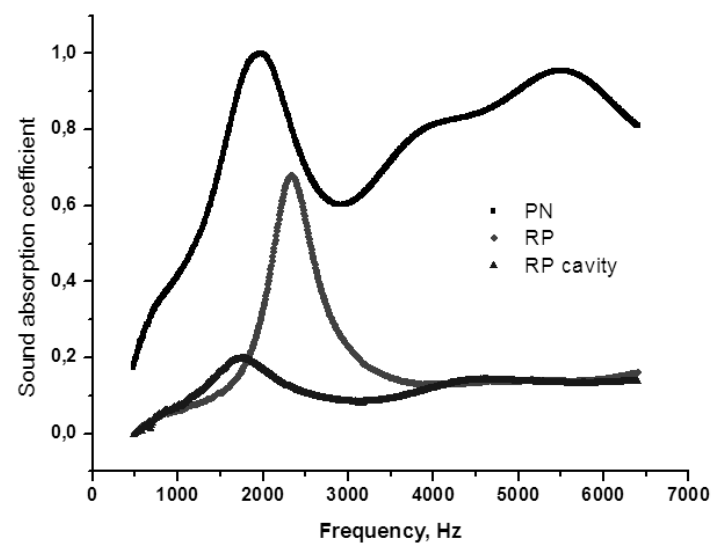

Fig. 5. The absorption coefficient for different samples

It is also clearly seen in Fig. 5 that the RP and RPcav samples have a low sound absorbing capabilities at high frequencies. It can be explained as follows; when a sound wave is transmitted into the RP, the sound waves are reflected and refracted causing a damping phenomenon that uses less energy and reduces heat loss, which finally results in a low sound absorption coefficient at high frequencies. This result also explains why the sound absorption coefficient of RP and RPcav samples is lower than the value obtained by the PN sample with the same pattern. The sample with a back cavity (RPcav) has the lowest coefficient of sound absorption (0.21), which indicate that the material is not appropriate as a sound absorbing the material. Sari et al., ${ }^{9}$ reported that sound absorption at lower frequencies over $1000 \mathrm{~Hz}-3000 \mathrm{~Hz}$ ) is desirable for automotive applications because this frequency range concurrences to the noise produced by the engine running, tires, and road, thereby making $\mathrm{NaHCO}_{3}$ - polyester absorber a promising candidate for the automotive interior sound absorber.

The real and imaginary parts of the acoustic impedance obtained from different samples are depicted in Fig. 6 and 7, respectively. The real parts are the barriers attributed with energy loss, and the imaginary part is the reactance related to phase change. In this case, we can see better PN sample performance than any other material that has been studied. It is clearly indicated that the increase of sodium bicarbonate content within the polyester resin decreases the impedance value of the absorbent sample. It implies that the decreasing of the impedance value will increase the fraction of the wave energy that can be transmitted into the material. The acoustic absorption coefficient for this type of absorber can be obtained from the resultant acoustic impedance of fibrous absorbent is expressed in the equations 1,2 and $3 .{ }^{10}$

$$
\begin{aligned}
& \alpha_{n}=\frac{4 \rho_{o} c_{o}\left(R_{p}+R_{1}\right)}{\left(\rho_{o} C_{o}+R_{p}+R_{1}\right)^{2}+\left(\omega M_{p}+M_{1}\right)^{2}} \\
& =\frac{4\left(r_{p}+r_{1}\right)}{\left(1+r_{p}+r_{1}\right)^{2}+\left(\omega m_{p}+m_{1}\right)^{2}} \\
& r_{p}=\frac{32 n t}{\varepsilon \rho_{o} C_{o} d^{2}} \sqrt{1+\frac{k^{2}}{32}} \\
& \omega m_{p}=\frac{\omega t}{\sigma C_{0}}\left(1+\left(3^{2}+\frac{k^{2}}{2}\right)^{-1 / 2}\right) \\
& k=d \sqrt{\frac{\omega \rho_{0}}{4 \eta}}
\end{aligned}
$$

Where poCo is the characteristic impedance of air, $\mathrm{k}$ is the constant of the perforated material, $\rho o=1.2 \mathrm{~kg} / \mathrm{m}^{3}, \mathrm{Co}=340 \mathrm{~m} / \mathrm{s}, \eta=1.85 \times 105 \mathrm{~kg} / \mathrm{s} / \mathrm{m}$. The RP, R1, MP, and M1 are the resistance and reactance of the perforated material, respectively, When the porosity of material $\varepsilon(\%)$, thickness of material $\mathrm{t}(\mathrm{mm})$, pores size $\mathrm{d}(\mathrm{mm})$ and frequency $f$ is known, the surface impedance of the material can be obtained by an impedance tube.

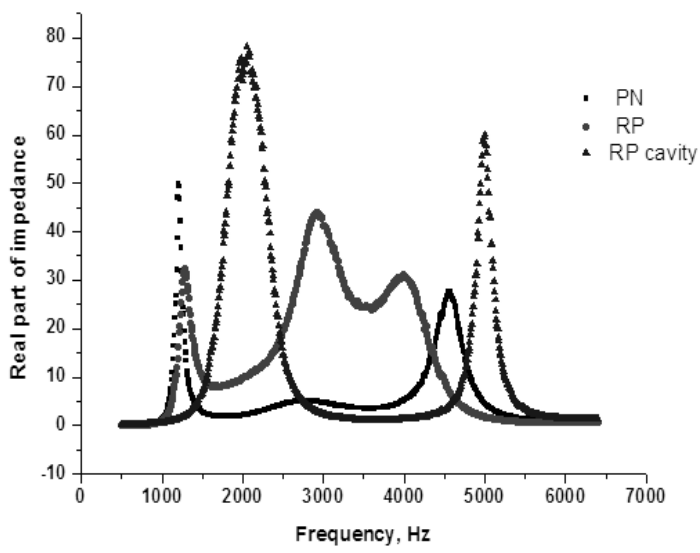

Fig. 6. The real part of the impedance ratio of different samples 


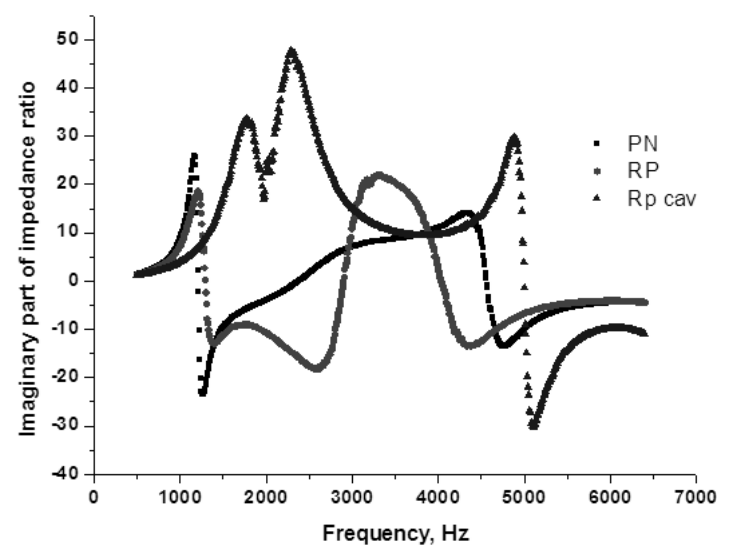

Fig. 7. The imaginary part of the impedance ratio of different samples

\section{CONCLUSION}

Based on the measurement results, it can be concluded that the sound absorption performance is greatly increased at low and medium frequencies in the presence of $\mathrm{NaHCO}_{3}$ filler in polyester resin. The use of back cavity reduced the sound absorption performance of materials at low and medium frequencies.

\section{ACKNOWLEDGMENT}

This reseach work is not geting any support from the institution and not geting fund from any funding agency.

\section{REFERENCES}

1. Sari, N. H.; Wardana, I. N. G.; Irawan, Y. S.; \& Siswanto, E. Corn Husk Fiber-Polyester Composites as Sound Absorber: Nonacoustical and Acoustical Properties. Advances in Acoustics and Vibration., 2018, 15(4), 545558. doi: 10.1080/15440478.2017.1349707.

2. Rodrigues, E. F.; Maia, T. F.; \& Mulinari, D. $R$. The tensile strength of polyester resin reinforced sugarcane bagasse fibers modified by esterification, Procedia Engineering, 2011, 10, 2348-2352. doi:10.1016/j.proeng. 2011.04.387.

3. Iwan, Y.; \& Harjana. New absorption improvement strategy for QRD element. In 20th International Congress on sound \& vibration. 2013, ICSV20. Bangkok, Thailand.

4. Xie, H.; \& Lyu, Y. Sound absorption characteristics of the perforated panel resonator with tube bundles. In The 21st International Congress on sound and vibration. 2014, ICSV 21, Beijing/China.

5. Gheorghe, A. Acoustic performances analysis of a composite system for noise barriers specific to road traffic. The Annals of DUNAREA DE JOS. 2013, University of Galati fascicle V. Technologies in machine building. ISSN 1221- 4566.

6. Chen, D.; Li, J.; \& Ren, J. Study on sound absorption property of ramie fiber reinforced poly (L-lactic acid) composites: Morphology and properties. Composites: Part A., 2010, 41,1012-1018. doi:10.1016/j.compositesa. 2010.04.007.

7. Jiang, S.; Xu, Y.; Zhang, H.; White, C. B.; \& Yan, X. Seven-hole hollow polyester fibers as reinforcement in sound absorption chlorinated polyethylene composites. Applied Acoustics., 2012, 73, 243-247. doi:10.1016/j.apacoust. 2011.09.006.

8. Shakhashiri. Sodium hydrogen carbonate and sodium carbonate. Chemistry. 2010, 104-2, www.scifun.org. Retrieved from http:// www.scifun.org/GenChem/CHEMWEEK/ Sodium $\% 20$ Bicarbonate $\% 20$ and $\% 20$ Sodium\%20Carbonate.pdf

9. Sari, N. H.; Wardana, I. N. G.; Irawan, Y. S.; \& Siswanto, E. Physical and Acoustical Properties of Corn Husk Fiber Panels. Advances in Acoustics and Vibration. 2016, Article ID 5971814, 8 pages. http://dx.doi. org/10.1155/2016/5971814

10. Maderuelo-Sanz, R.; Nadal-Sisbert, A. V.; Crespo-amorós, J. E.; \& Farres-Garcia, F. A novel sound absorber with recycled fibers coming from the end of life tires (ELTs). Applied Acoustics., 2012, 73(4), 402-408. doi:10.1016/j.apacoust.2011.12.001. 